\title{
Formation of a Junior Doctor Wellbeing Steering Group
}

\author{
Author: Amy Davies*
}

\section{Introduction}

Reports by the British Medical Association, Royal College of Physicians and Health Education England have all raised concerns regarding the wellbeing of junior doctors. Working in an increasingly demanding, poorly resourced and underfunded healthcare system is associated with a triad of guilt, low selfesteem and sense of failure. ' Understandably, this not only affects the day-to-day working of individuals, but also extends detrimentally to affect home life and mental health.

Wellbeing initiatives are numerous and of varying success. What is recognised is that there is an appetite for change and hospitals need to prioritise the development of a wellbeing culture within their workforce.

The setting of our steering group is a newly built, world-renowned large tertiary teaching hospital, which often attracts doctors in training specifically to develop specialty interests. Despite these positive elements, anecdotally and also from junior doctor surveys, the hospital is often described as impersonal and junior doctors commonly describe feeling like a 'cog in a machine'.

\section{Methods}

A primary scoping exercise of previous wellbeing initiatives indicated that they were either individually led or as a result of a small groups working separately within the hospital. The aim of the Wellbeing Steering Group was to initially bring these individuals together to enable collaborative working. At this stage these individuals were predominantly consultants. Invitations were then extended to members of the education team, junior doctor support team, occupational health, nursing colleagues and junior doctor representatives.

\section{Results}

There are currently 18 members of the Junior Doctor Wellbeing Steering Group. Meetings are currently held quarterly. The aims of the meetings are threefold.

$>$ Introduce like-minded individuals passionate about junior doctor wellbeing.

$>$ Encourage discussion regarding the development of existing initiatives and a non-judgmental forum to discuss ideas for future initiatives.

Author: University Hospitals Birmingham NHS Foundation Trust ${ }^{*} \mathrm{RCP}$ chief registrar
> Ensure that smaller working groups are allocated to specific initiatives to enable work to be completed and fed back to all members of the steering group.

A summary of each meeting is emailed out to all members after large group meetings, with updates in between to ensure all are up to date with developments.

Ongoing initiatives include:

> Small group reflective practice groups facilitated by a consultant psychologist to discuss difficulties faced as a junior doctor.

> Implementation of Schwartz rounds (multiprofessional reflective practice).

> Development of a 'buddy system' for new doctors joining the hospital.

> Encouraging positive feedback eg promoting the use of the 'Learning from Excellence' scheme.

\section{Conclusion}

A single junior doctor wellbeing initiative is unlikely to be successful in isolation. Although in its infancy, the Junior Doctor Wellbeing Steering Group provides a forum for passionate individuals advocating the importance of junior doctor wellbeing and is a much-needed positive step forward. Collaborative working allows ideas to be developed simultaneously with the hope that we can increase the number of initiatives and have a positive impact on junior doctor wellbeing.

\section{Reference}

1 Gabbard G. The role of compulsiveness in the normal physician. JAMA 1985;254:2926-9. 